\title{
Autism Spectrum Disorder: Patients' Oral Health Behaviors and Barriers in Oral Care from Parents' Perspectives
}

\author{
Maryani Mohamed Rohani ${ }^{1 *}$, Nur Fatin Baharozaman ${ }^{1}$, Nur Syadiyah Khalid ${ }^{1}$, \\ Norintan Ab-Murat ${ }^{2}$
}

KEYWORDS

oral health, barriers to care, autism

\begin{abstract}
To assess the oral health behaviors of children with Autism Spectrum Disorder (ASD) and explore attitudes and barriers in providing oral care by their parents. A cross-sectional, mixed method study design, which comprised of quantitative and qualitative sections was conducted. The quantitative part assessed the oral health behavior through a parent-proxy report questionnaire whilst the qualitative part assessed parents' overall perspective on maintenance of oral health in children with ASD through an in-depth interview using semi-structured questions. All children below the age of 16 who have been clinically diagnosed with ASD and registered at the Pediatric Dental Clinic were included. The sample consisted of twenty children with ASD. Most of the sample were male and between 5-8 years old. Majority had good oral health behaviors but some exhibited self-injurious behaviors that may affect oral health. Severe characteristics of ASD, co-morbid conditions and incompetent health professionals were reported as barriers in oral care by parents of these children. Despite having good oral health behaviors, several barriers and challenges were expressed by these parents with ASD when providing home oral care or bringing them for dental appointment. Continuous support for parents with ASD child by dental professionals is essential to help overcome the real challenges and barriers in providing oral care among these parents.
\end{abstract}

\section{INTRODUCTION}

Autism Spectrum Disorder (ASD) cases are becoming more common since the last few decades due to the growing awareness of ASD among parents, clinicians and researchers. It is a spectrum of pervasive developmental disorders that consists of Autistic Disorder, Asperger's Disorder and Pervasive Developmental Disorder Not Otherwise Specified (PDD-NOS) [1]. Studies in Asia, Europe, and North America have identified

\footnotetext{
1 Department of Pediatric Dentistry and Orthodontics

2 Department of Community Oral Health and Clinical Prevention Faculty of Dentistry, University of Malaya, Kuala Lumpur, Malaysia.

* Correspondence: drnani@um.edu.my
}

individuals with ASD with an average prevalence of between $1 \%$ and $2 \%$ [2]. In Malaysia, there is no official report stating the prevalence of ASD among its children [3]. This may be partly due to the absence of official registry specific for the condition as they are grouped together with other type of disabilities [4]. However, based on the Modified Checklist for Autism in Toddlers (MCHAT) by the Ministry of Health Malaysia, it is estimated that about 1.6 in 1000 Malaysian children have ASD [3].

ASD is characterized by three definite criteria; disability to carry out a reciprocal social interaction, impaired social communication of either verbal or non-verbal and repetitive interests or behavior [5]. Commonly, children with ASD are diagnosed during the first three years of their lives, 
with a higher predilection in males, four times more than females [5]. Amongst those children with recorded IQ scores, about a third also had intellectual disability of IQ score less than 70 [6].

Individuals with ASD have special characteristics that may have significant impact on their oral health behaviors [7]. Most of them have impaired reciprocal social interaction with poor eye contact that cause difficulties for parents or dentists to provide oral care [5]. For example, their fine motor control is usually impaired, thus assistance from their parents or caregivers during tooth brushing is often required [8]. In addition, these children may also have hypo or hyper-sensitive to sensory stimuli such as unfamiliar taste of toothbrush or toothpaste, loud noises and bright lights $[5,8,9]$. In order to suppress the ASD-related behaviors, some of these children are also prescribed with antipsychotic medications that may cause dry mouth and gingival enlargement $[8,10,11]$. Due to these circumstances, parents need to be motivated and be more attentive when providing oral care to ensure maintenance of oral health [7].

Most children with ASD are unable to express their feelings using spoken language, gestures or eye contact, thus they are not able to communicate their complaints as compared to other children without ASD [5]. They are more inclined towards repetitive interests or behavior and may refuse to accept any new changes in their daily routine [5]. Unfamiliar visit to a dental clinic and treatment from a dentist may be aggressively objected [5]. Poor cooperation during dental visit has been linked to their dislike to foreign environment, physical contact and stimuli such as bright light and loud sounds [8]. Hence, dental visits can be challenging for them and parents have been found to be uncomfortable with taking their child with ASD to the dentist [7]. Parents' willingness to bring their child to dentists has also been linked to the severity of their child's ASD conditions $[7,8]$. Therefore, it is incumbent to incorporate oral hygiene practices and dental visits as part of their routine in order for these children to adapt to such behaviors [7,12].

Other common behaviors of children with ASD which may affect the provision of dental care include temper tantrums, hyperactivity, short attention span, impulsivity, agitation, anger and self-injurious behaviors (SIBs) [7]. To control the aggressive behaviors of children with ASD, sweet foods are usually given as a reward and some have suggested that individuals with ASD may actually prefer sweet foods [12]. However, some studies have indicated that children with ASD are less partial to sugary foods [7] and this may possibly explain their caries rate which is comparable to the normal population $[8,13,14]$.

It is important to assess the oral health behavior of children with ASD as this have a significant impact on their overall oral health status. Parents' perceptions towards providing oral care to children with ASD should be explored as these children may need to depend on their parents in achieving or maintaining good oral health. This study was conducted with the purpose to assess the oral health behavior of selected children with ASD and to identify parental barriers in providing oral care to their children with ASD. The findings of this study will enhance understanding and awareness to both parents and oral health professionals so that improved oral health care for these children can be delivered.

\section{MATERIALS AND METHODS}

\section{Study design}

This was a cross-sectional, mixed-method study design, which comprised of quantitative and qualitative sections. The quantitative part assessed the oral health behaviors of children with ASD, while the qualitative part assessed parents' perceived barriers of providing oral care to their child with ASD. The triangulation mixed mode design was employed to obtain different but complementary data on the same topic [15]. This study was approved by the University of Malaya Ethics Committee (DF CO1603/0019(U)) and the Medical Research \& Ethics Committee (NMRR-16955-29876 (IIR).

\section{Study participants}

All children below the age of 16 who have been clinically diagnosed with ASD and registered the Pediatric Dental Clinic at Faculty of Dentistry, University of Malaya were the reference population for this study. The name list and their respective medical history and contact details were obtained from the patients' digital database system. Parents or caregivers of these children with ASD were then personally contacted via telephone. All parents who participated in the quantitative part were invited to participate in the qualitative part of the study.

\section{Study instruments}

Data for the quantitative part was collected through a parent-proxy report questionnaire. The questionnaire was constructed based on a 
literature review [7] and discussion with experts from the Special Care Dentistry and Dental Public Health fields. It consisted of questions on demographic background of parents and children, oral health behavior variables which include tooth brushing, flossing, sugar intake and dental attendance, and questions on self-injurious behaviors and factors affecting dental visits. All items in the questionnaire underwent a face and content validation by three senior academics who are familiar with the topic content. The questionnaire was then pilot-tested on five parents with a child below the age of 16 years attending the Pediatric Dentistry Clinic. No modification was made on the questionnaire as the feedback received was positive.

Parents who agreed to participate in the quantitative survey were given an appointment date at the Pediatric Dental Clinic. Apart from receiving dental check-up for their children during the visit, parents were requested and assisted to complete the parent-proxy questionnaire. All parents or caregivers who took part in the quantitative study were invited to take part in the qualitative section conducted by two interviewers (NFB and NSK), which took place at their own residences. The interview took about $1.5-2.5$ hours. Prior to the interview, consent was taken from the parents for recording of conversation using a digital recorder. We used the general interview guide approach (or a guided interview) where a basic checklist is prepared to make sure that all relevant topics were covered. However, the interviewer was still free to explore, probe and ask questions deemed interesting to the researcher, until a saturation of data was achieved. The checklist or semi-structured questions used in the interview were designed based on literature reviews and covered specific areas regarding the barriers and challenges in providing oral care to children with ASD. Among the questions asked were the difficulties in brushing their children's teeth and challenges in terms of time and energy in providing oral care.

\section{Data analysis}

The quantitative data were analyzed using Statistical Package for Social Sciences (SPSS Incorporated, Chicago, Version 12.0 Illinois, USA) software for Windows. Descriptive statistic such as frequencies and percentages were computed to report on the findings. For the qualitative part, the audiotaped interviews were transcribed using verbatim and the content were analyzed by performing thematic analysis. All the transcripts were read, and open coding were performed by two researchers. Open coding involved assigning codes line by line to form initial coding scheme. Then, related codes were clustered together to identify themes. The identified themes were then evaluated and discussed until agreement was reached. For reporting purpose, quotes in Malay language were translated to English.

\section{RESULTS}

\section{Part 1: Quantitative Data}

Based on the patients' digital database system, there were 27 children with ASD registered at the Pediatric Dentistry Clinic. Of these, only 25 of the records had valid contact information. However, upon contacting the patients or their parents, only 20 agreed to participate, giving a response rate of $80 \%$. Those who declined stated time constraints as the main reason for not participating.

Most children with ASD who participated in this study were boys (80\%) and were attending school (90\%) (Table 1). Slightly more than half were Malays and about one-fourth were Chinese. Half of them were in the 4-8 age group. Most of the children in this study were diagnosed with Autistic Disorder (85\%), while only two had PDD-NOS condition. Half of the children were from a family with a household monthly income of between RM5,000-RM10,000.

Table 2 describes the oral health behavior of the selected children with ASD.

About three-quarter of them brushed teeth more than once a day and all reported using a manual toothbrush. However, one child never had their teeth brushed at all. Concerning their brushing habit, most children used fluoridated toothpaste $(60 \%)$ and required assistance from their parents during tooth brushing rituals (60\%). When inquired about other oral hygiene aids used, only a quarter of them reported using other aids such as cloth, cotton bud and gauze, as a supplementary to tooth brushing. On the sugary diet aspect, only less than one-fifths was given sugar rewards by their parents during temper tantrum episodes. With regards to dental attendance, most children had their last dental visits less than six-months ago (70\%), and the reasons were mainly for a normal check-up (45\%). Half of the parents reported that noisy equipment and unfamiliar sensation were the main factor that could affect their child's anxiety and cooperation during dental visits (Table 3). 
Table 1. Demographic characteristics of selected children with ASD and their parents $(N=20)$

\begin{tabular}{ll}
\hline Background & $\mathrm{N}(\%)$ \\
\hline Sex & \\
Boys & $16(80)$ \\
Girls & $4(20)$ \\
\hline Age & \\
0-4 years old & $1(5)$ \\
5-8 years old & $10(50)$ \\
9-13 years old & $9(45)$ \\
\hline Ethnicity & \\
Malay & $11(55)$ \\
Chinese & $5(25)$ \\
Indian & $2(10)$ \\
Others & $2(10)$ \\
\hline Attending school & \\
Yes & $19(95)$ \\
No & $1(5)$ \\
\hline Autism spectrum disorders & \\
Autistic disorder & $17(85)$ \\
Asperger syndrome & $0(0)$ \\
PDD-NOS & \\
\hline Do not know & \\
\hline Rarental average & \\
\hline
\end{tabular}

\section{PART 2: QUALITATIVE DATA}

The following findings were obtained from nine parents who agreed to participate in the in-depth interview. Eleven parents were not willing to participate due to personal reasons. Following the analysis of the transcribed data, three themes emerged relating to parental barriers on providing oral care to their child with ASD. Other factors include child's challenging behavior (10\%), child dislike physical contact (10\%) and hospital-like environment (5\%). Table 4 describes the behaviors exhibited by children with ASD that may indirectly affect their oral health. A quarter of these children liked to bite on soft object and a lower percentage liked to bite on hard objects and clench/grind their teeth.
Table 2. Oral health behaviors among selected children with ASD $(\mathrm{N}=20)$

\begin{tabular}{|c|c|}
\hline Oral Health Behaviours & $\mathbf{N}(\%)$ \\
\hline \multicolumn{2}{|l|}{ Frequency of brushing } \\
\hline More than once a day & $15(75)$ \\
\hline At least once a day & $4(20)$ \\
\hline Never & $1(5)$ \\
\hline \multicolumn{2}{|l|}{ Type of toothbrush used } \\
\hline Manual toothbrush & $20(100)$ \\
\hline \multicolumn{2}{|l|}{ Type of toothpaste used } \\
\hline Fluoridated & $12(60)$ \\
\hline Non-fluoridated & $5(25)$ \\
\hline Not sure & $3(15)$ \\
\hline \multicolumn{2}{|l|}{ Tooth brushing assistance } \\
\hline Assisted by parents/caregiver & $12(60)$ \\
\hline Supervised by parents/caregiver & $5(25)$ \\
\hline On their own & $2(10)$ \\
\hline Do not brush & $1(5)$ \\
\hline \multicolumn{2}{|l|}{ Tooth brushing aids used } \\
\hline Floss & $0(0)$ \\
\hline Mouthwash & $1(5)$ \\
\hline $\begin{array}{l}\text { Others (towels, cotton bud, gauze, } \\
\text { electric toothbrush) }\end{array}$ & $5(25)$ \\
\hline None & $15(75)$ \\
\hline
\end{tabular}

These themes are: (1) Children's personal factors that affect tolerance towards oral health care, (2) parental attitude toward oral care of their child with ASD, and (3) parents' personal experiences after previous dental visits with their child with ASD (Figure 1).

$\begin{array}{lll}\begin{array}{l}\text { Factors } \\ \text { affect }\end{array} & \begin{array}{l}\text { Parents' personal } \\ \text { experiences after } \\ \text { previous dental visits }\end{array} & \\ \begin{array}{l}\text { i)Medical } \\ \text { condition }\end{array} & \begin{array}{l}\text { i)Improved tolerance } \\ \text { ii)Better compliant }\end{array} & \begin{array}{l}\text { Parental } \\ \text { attitude }\end{array} \\ \begin{array}{l}\text { ii)Need } \\ \text { asistance }\end{array} & \begin{array}{l}\text { iii)Skills, patience, } \\ \text { experience, knowledge }\end{array} & \begin{array}{l}\text { i)Positive } \\ \text { attitude }\end{array} \\ \begin{array}{l}\text { iii)Lack } \\ \text { tolerance }\end{array} & & \begin{array}{l}\text { ii) Negative } \\ \text { attitude }\end{array} \\ \text { iv)Special } & & \\ \text { school } & \text { Parents' } & \\ & \text { perspective } & \end{array}$

Figure 1. Parents' perspective on ASD and oral health care 
Theme 1: Children's personal and environmental factors that affect tolerance towards oral health care

\section{Subtheme 1: Associated medical conditions}

Parents associated their children's accompanying medical conditions with difficulties in maintaining oral hygiene care. Some children were diagnosed with other medical disorders during infancy, which may affect children's intellectual or physical capacity to undergo daily oral hygiene care in an effective manner. As a result, parents had more difficulties in providing oral care regimen at home, as reported by these parents:

Table 3. Factors that affects anxiety or decrease cooperation in child with ASD during dental visit.

\begin{tabular}{ll}
\hline $\begin{array}{l}\text { Factors that may increase anxiety } \\
\text { or decrease cooperation* }\end{array}$ & $\mathbf{N}(\%)$ \\
\hline Noisy equipment & $10(50)$ \\
\hline Unfamiliar sensation & $10(50)$ \\
\hline Bright light & $3(15)$ \\
\hline Others & $1(5)$ \\
Parents did not give attention & $2(10)$ \\
Child dislike physical contact & $1(5)$ \\
Hospital-like environment & $2(10)$ \\
Challenging-behaviour & $1(5)$ \\
$\begin{array}{l}\text { Inexperience dentist in treating a } \\
\text { child with ASD }\end{array}$ &
\end{tabular}

*Multiple answers were allowed for the question

Table 4. Self-injurious behaviours exhibit by children with ASD that may affect oral health

\begin{tabular}{ll}
\hline Self-injurious behaviours* & N (\%) \\
\hline Biting on soft object & $5(25)$ \\
\hline Biting on hard object & $4(20)$ \\
\hline Self-injury & $3(15)$ \\
\hline Clenching/grinding teeth & $4(20)$ \\
\hline Hand-flapping & $5(25)$ \\
\hline Others & \\
Jumping & $3(15)$ \\
Nail biting & $2(10)$ \\
Throwing tantrum & $2(10)$ \\
None & $5(25)$ \\
\hline
\end{tabular}

*Multiple answers were allowed for the question
Parent 1: "She was diagnosed with tuberous sclerosis in the brain when she was 6 months old. She was then diagnosed with ASD as the illness gave rise to $A S D$ behaviors. Oral care is challenging as she could not understand the purpose of brushing and comprehend the importance of it."

Parent 5: "He was diagnosed with autism in 2013 and with Hunter Syndrome in 2014. One of the syndrome manifestations was joint stiffness. So, he cannot even hold the toothbrush properly."

\section{Subtheme 2: Assistance in basic daily tasks}

Parents also reported that their child with ASD required more attention and needs compared to the other healthy children. Parents had to help their child with basic daily tasks, such as feeding, drinking and bathing. Therefore, oral hygiene care had the least priority, as explained by this parent:

Parent 5: "Due to his condition, we have to do a lot of things for him. So, the checklist is long and brushing teeth comes at the very end. Since both of us are working, by the time we completed the entire checklist, we wear ourselves out."

Subtheme 3: Lack of tolerance with behavioral difficulty

Parents commented that it was difficult to place toothbrush in their child's mouth as the child with ASD will never tolerate the objects that are disliked by them. These parents reported that the child:

Parent 3: "...bites on the toothbrush firmly and he will do anything to prevent me from brushing her teeth"

Parent 6: "... will get mad when I try to brush his teeth. The more I try to force him, the more he will refuse. He would bite the toothbrush the moment it is in his mouth."

Most parents reported that their child's challenging behavior became worst especially during their first dental appointment. One parent expressed that the difficulty increased as the child grew. This was further described by these parents:

Parent 7: "He struggled a lot and did not give any cooperation during the first dental visit. They only managed to do a simple check-up and did not carry out any treatment."

Parent 9: "During the first dental visit he refused to open his mouth and struggled a lot and we had to hold him. He also knocked all the dental equipment everywhere. Sometimes, I would lie on the dental chair and hold him but now it is even more difficult 
since he is bigger and stronger."

\section{Subtheme 4: Special education school}

Most parents sent their children with ASD to occupational therapies or special needs school and reported a change in the child's social interaction. These parents described that:

Parent 4: "I can see changes when he started school. He started to communicate with other people. So, he can understand when I explain about why I need to brush his teeth."

Parent 7: "....... after undergoing therapy, now he is a bit stable and able to sit on the dental chair for a while."

\section{Theme 2: Parental attitude towards oral care among children with ASD}

\section{Subtheme 1: Positive oral-health attitude}

Most parents relayed their struggles in trying to brush their child's teeth. Some addressed the difficulties by trying different strategies to tailor to their own child's needs and ensure that their teeth were brushed. Some of the comments were:

Parent 1: "I tried to attract her attention by buying fancy toothbrush."

Parent 5: "He does not know how to spit out after brushing. So, I usually wipe the inside of his mouth to remove the residual toothpaste so that he would not swallow the fluoride in the toothpaste."

Some parents demonstrated admirable attitude in ensuring their child's oral health was optimal, although their time and energy was claimed to be insufficient, as these parents commented that:

Parents 2: "I will make sure I brush his teeth especially before bed even if I am too tired."

Parents 9: "I will not skip his regular brushing time. For example, even though he has slept, I will still make him brush his teeth."

\section{Subtheme 2: Negative oral-health attitude}

Oral health was not part of their child's daily health routine and this attitude was reflected by one of the parents who admitted to only start to brush her child's teeth after being advised by the dentist at a late age. She also confessed to almost giving up in performing oral care for the child due to the child's challenging behavior. The same parent admitted to be a symptomatic dental attender as she does not go for a regular dental check-up due to time constraint and cost. This parent's behavior reflected on the provision of care to her child with ASD, as commented by:

Parent 6: "I only started to brush his teeth after his first appointment with the dentist when he was 7 years old. But, whenever I put the toothbrush in his mouth, he would bite it. So, I do not brush his teeth until now. Perhaps it would be easier when he is older".

\section{Theme 3: Parents' personal experiences after previous dental visits with their child with ASD}

Most parents reported that dental visit was helpful in improving the child's oral health through oral health education received during dental visit and the child was able to tolerate better during oral care, as stated by:

Parent 4: "It was a struggle to brush his teeth before. But after I met a dentist at the hospital, he taught me how to brush the back teeth. My child is now more cooperative during tooth brushing."

Another parent mentioned that their child was able to comprehend the consequences of not maintaining good oral hygiene after receiving dental treatment. The child was more compliant in performing oral care at home as well as during dental visit, as stated by:

Parent 3: "He had previous experience of losing 10 teeth. Since then, he has taken good care of his teeth because he does not want to lose his teeth again."

It was difficult, as remarked by some of the parents, to bring their child for dental visits because they were traumatized from the previous bad experiences due to insufficient knowledge and skills of oral health professionals in managing patients with ASD and their lack of understanding in ASD-related behaviors, as indicated below:

Parent 8: "He does not tolerate well whenever we go to the hospital because he had very bad experiences as the staff do not know how to handle him. So, the more the staff forced him, the worst his tantrum will be."

Parent 9: "Most dentists do not have the patience to treat autism kids and they are also scared of them. I think we should have more well-trained dentists or dental therapists in managing special need kids."

\section{DISCUSSION}

Most children with ASD have impaired communication and social interactions that can have significant impact on their oral health behaviors, including tooth brushing and flossing [6]. However, it is interesting to note that most of the children with ASD in our study had a relatively good oral behavior as most them brushed their 
teeth as part of their daily routine, used fluoridated toothpaste and had low intake of sugar. This positive result could possibly be because the participants attended the Pediatric Dental Clinic and had better awareness about oral health as reported in other studies $[16,17]$. In addition, the quantitative part was based on the interview with the parents about their children (parent-proxy measure) and hence the accuracy or truthfulness of the answer was questionable.

We also found that most of the children in in this study required assistance from their parents during brushing that could partly be due to the degree of severity in ASD. This finding should also be interpreted with caution as most of younger aged children generally require some assistance and monitoring when brushing compared to the older age groups.

Even though most of the children had good oral health behavior, parents shared the problems they faced in performing oral care for their children with ASD in the qualitative part of the study. Parents who have children with ASD with other underlying medical problems expressed more difficulties in trying to care for their child's oral health as the child would have a more severe cognitive disability and behavioral problems. Some medical conditions also affect their musculoskeletal function which results in poor manual dexterity in performing oral hygiene routines by themselves $[7,20]$. These difficulties may demotivate parents to ensure continuous oral care to their child with ASD. It is very critical that children with ASD perform effective oral hygiene procedures as they were found to have more debris and calculus deposits than the general child population [8,21]. A method of visual pedagogy has been found to aid in teaching and encouraging children with ASD to brush their teeth [20]. Since children with ASD have a tendency to decline a new routine such as tooth brushing, parents might have to consider this technique in order to familiarize their children with the ritual. This method includes a series of pictures that shows tooth brushing steps and techniques that can be placed in a bathroom or wherever tooth brushing activity is commonly performed at home. Apart from that, dental care providers should also cater support to the parents as they play an important role in supervising the children's oral hygiene activities. These efforts include preparation of parents' education material and guidance in oral health related knowledge [6].

Apart from the difficulties of performing home oral hygiene care, dental visits were challenging for some parents in this study. This finding was similar to those reported by Lewis et al. where most parents in his study stated that waiting in the dental clinic was a very stressful event as their child with ASD became more anxious and agitated

[23]. In our qualitative part, noisy equipment and unfamiliar sensation were reported to increase anxiety or decrease cooperation of their child in dental clinic. Other factors such as child's challenging behavior, physical contact and hospital-like environment may also affect their cooperation. Studies conducted on children with ASD confirmed that they are hypersensitive to various stimuli such as sounds, lights, odors, colors [8] and physical contact [24] hence making oral examination in a dental clinic a difficult task for the oral health professionals [22]. Children with ASD also dislike any changes in their routine, making them unable to respond positively in a new environment such as unfamiliar dental clinic and staffs [8]. Hence, preparatory visit is important in order to increase their cooperation. To ensure some familiarity to these children, dental examination should be conducted in the presence of their parents $[8,25]$. Some parents in our qualitative section also described that the first dental visit for their children with ASD was very challenging as they did not give any cooperation and refused to open their mouth. It is recommended that oral care providers explain the nature of any dental procedures to be performed to both parents and child with the help of pictures [8]. Tell-show-do techniques and short, clear instruction as well as positive verbal reinforcement can be used to increase compliance of the children with ASD in the dental clinic [26].

When parents in this study were asked to indicate any other barriers that could affect proper oral care in the dental clinic, inexperience dentists and dental therapists were stated as one of the barriers. This issue needs to be addressed as studies have reported that children with ASD have more unmet dental needs than the normal children $[9,27,28]$. Parents with children with ASD in this study also demanded a proper training of healthcare professionals in handling children with ASD, as well as the other types of special needs children. Lack of exposure in clinical and didactic teaching amongst dental undergraduates [29-34] were among many other reasons of why there was a lack of consideration to providing dental care and treatment for people with special needs [35]. Ideally, based on a spectrum of need and disability [36], specialists in Special Care Dentistry (SCD) 
have a role to play in managing individuals with the most severe and profound disability compared to those with mild to moderate disability. The latter only require specialist dental care when additional support or expert opinions are required. Increased education, exposure, and training in managing patients with special needs at dental school can improve the general dentist skills in managing patients with mild to moderate disability [31]. When there are more general dentists who are well trained to treat these patients, a large cohort of them can access professional dental services. Since there is a lack of SCD specialists available to cater for the high treatment needs, it is important for the general dentists to be able to manage these patients, which also then help to reduce less inappropriate referrals to the specialist $[19,37]$. The incorporation of SCD in dental courses in some countries, for example in Malaysia and Australia, seems to be the remedy for this lack of education in this specialty, however, the number of the experts to drive SCD education is still not adequate [38].

The findings of our study should be interpreted with cautions. Our samples consisted of children with ASD who patients at the Pediatric Dental Clinic were and hence the good oral behavior that they have may be due to exposure to oral health education received during their treatment. Different findings could be obtained if samples were taken from non-hospital-based settings such as special needs centers or private homes. The sample size was small; however, there were only 27 children with ASD registered at the study location. This registered number of patients with ASD was confirmed by the head of the Pediatric Dental Clinic (Special Needs Dentistry Division) who also assisted in screening for potential samples. As there is no specific medical test that can diagnose autism and dental personnel may be unfamiliar with diagnosing autism, patients' medical record at this clinic was based only on parents' self-report of their children's medical condition. Hence, the number may not be accurate as some parents may not be aware that their child has ASD.

As the number of individuals with ASD is increasing with highly unmet dental needs (28), there is a need for our oral health professionals to be equipped with the necessary knowledge and skills to ensure effective provision of oral care to these populations. Appropriate support and encouragement should also be provided to the parents to motivate them in continuing oral care at home. We recommend that future studies should include samples from different settings and assess the oral health behavior of children with different severity level of ASD. Also, there has not been any mixed method study conducted on the similar issues, hence our study may serve as a reference for future related studies.

\section{CONCLUSION}

Most of the children with ASD in our study who were patients at the Pediatric Clinic had good oral health behaviors especially pertaining to their tooth brushing habit, usage of fluoride toothpaste, sugary intake and attendance at dental clinics. However, their parents expressed several barriers and challenges when trying to provide home oral care for these children or bringing them for a dental visit.

\section{REFERENCES}

1. APA. Diagnositic and statistical manual of mental disorders, text revision (4th ed.). Washington, DC : American Psychiatry Association; 2000.

2. Centers for Disease Control and Prevention (CDC). Autism Spectrum Disorder (ASD). Data and Statistics. [cited 2018 Aug 1]. Available from: https://www.cdc.gov/ncbddd/autism/data.html

3. Clinical Practice Guidelines Management of Autism Spectrum Disorder in Children and Adolescents. Ministry of Health Malaysia [Internet]. Malaysian Health Technology Assessment Section (MaHTAS); 2014 Apr 30;:1-76. Available from: http://www.moh.gov.my

4. Neik, T., Lee, L.W., Low, H.M., Chia, N. Prevalence, Diagnosis, Treatment and Research on Autism Spectrum Disorders (ASD) in Singapore and Malaysia. International Journal of Special Education, 2014; 27:1-10.

5. Dougall, A., Fiske, J. Access to special care dentistry, part 2. Communication. Nature Publishing Group. 2008;205(1):11-21.

6. Autism and Health: A Special Report by Autism Speaks [Internet]. www.autismspeaks.org. 2017. pp. 137. Available from: https://www.autismspeaks.org/science-news/autism-and-health-special-reportautism-speaks

7. Weil, T.N., Inglehart, M.R. Three-to 21-year-old patients with autism spectrum disorders: parents' 
perceptions of severity of symptoms, oral health, and oral health-related behavior. Pediatric Dentistry. 2012; 10:1-7.

8. Gupta, M. Oral health status and dental management considerations in autism. Int J Contemp Dent Med Rev. 2014. Article ID 021114

9. Stein, L.I., Polido, J.C., Mailloux, Z., Coleman, G.G., Cermak, S.A. Oral care and sensory sensitivities in children with autism spectrum disorders. Spec Care Dentist. 2011;31(3):102-10.

10. Murshid, E.Z. Oral health status, dental needs, habits and behavioral attitude towards dental treatment of a group of autistic children in Riyadh, Saudi Arabia. Saudi Dent J. 2005. 13(1):

11.Jaber, M.A. Dental caries experience, oral health status and treatment needs of dental patients with autism. J Appl Oral Sci. 2011;19(3):212-7.

12. Waldman, H.B., Perlman, S.P., Wong, A. Providing dental care for the patient with autism. J California Dental Association. 2008;36(9):662-70.

13. Scully, C., Dios, P.D., Kumar, N. Special Care in Dentistry E-Book: Handbook of Oral Healthcare. 2006.

14.Loo, C.Y., Graham, R.M., Hughes, C.V. The Caries Experience and Behavior of Dental Patients With Autism Spectrum Disorder. The Journal of the American Dental Association. 2008;139(11):1518-24.

15. Shapira, J., Mann, J., Tamari, I., Mester, R., Knobler, H., Yoeli, Y., et al. Oral health status and dental needs of an autistic population of children and young adults. Spec Care Dentist. 1989; 9(2):38-41.

16. McKinney, C.M., Nelson, T., Scott, J.M., Heaton, L.J., Vaughn, M.G., Lewis, C.W. Predictors of Unmet Dental Need in Children With Autism Spectrum Disorder: Results From A National Sample. Academic Pediatrics. Elsevier Ltd; 2014 Nov 12;14(6):624-31.Morse JM. Approaches to qualitative-quantitative methodological triangulation. Nurs Res. 1991 Mar;40(2):120-3.

17. Delli, K., Reichart, P.A., Bornstein, M.M., Livas, C. Management of children with autism spectrum disorder in the dental setting: Concerns, behavioural approaches and recommendations. Med Oral. 2013;e862-8.

18. Khatib El, A.A., Tekeya El, M.M., Tantawi El, M.A., Omar, T. Oral health status and behaviours of children with Autism Spectrum Disorder: a case-control study. International Journal of Paediatric Dentistry. 2014;24(4):314-23.

19. Mohamed Rohani, M., Calache, H., Borromeo, G.L. Referral patterns of special needs patients at the Royal Dental Hospital of Melbourne, Victoria, Australia. Australian Dental Journal. 2017;62(2):173-9.

20. Madore, C., Leyrolle, Q., Lacabanne, C., Benmamar-Badel, A., Joffre, C., Nadjar, A. et al. Neuroinflammation in Autism: Plausible Role of Maternal Inflammation, Dietary Omega 3, and Microbiota. Neural Plasticity. 2016;2016:3597209.

21. Subramaniam P, Gupta M. Oral Health Status of Autistic Children in India. Journal of Clinical Pediatric Dentistry, 2011;36(1):43-7.

22. Pilebro, C., Bäckman, B. Teaching oral hygiene to children with autism. International Journal of Paediatric Dentistry. 2005;15(1):1-9.

23. Lewis, C., Vigo, L., Novak, L., Klein, E.J. Listening to Parents: A Qualitative Look at the Dental and Oral Care Experiences of Children with Autism Spectrum Disorder. Pediatr Dent. 2015;37(7):E98-104.

24. Rogers, S.J., Hepburn, S., Wehner, E. Parent reports of sensory symptoms in toddlers with autism and those with other developmental disorders. J Autism Dev Disord. 2003;33(6):631-42.

25. Friedlander, A.H., Yagiela, J.A., Paterno, V.I., Mahler, M.E. The neuropathology, medical management and dental implications of autism. J Am Dent Assoc. 2006;137(11):1517-27.

26. Kamen, S., Skier, J. Dental management of the autistic child. Spec Care Dentist. 1985;5(1):20-3.

27. Desai, M., Messer, L.B., Calache, H. A study of the dental treatment needs of children with disabilities in Melbourne, Australia. Australian Dental Journal. 2001;46(1):41-50.

28. Lai, B., Milano, M., Roberts, M.W., Hooper, S.R. Unmet Dental Needs and Barriers to Dental Care Among Children with Autism Spectrum Disorders. J Autism Dev Disord. 2011;42(7):1294-303.

29. Weil, T.N., Inglehart, M.R. Dental education and dentists' attitudes and behavior concerning patients with autism. Journal of Dental Education. 2010;74(12):1294-307

30.Dao, L.P., Zwetchkenbaum, S., Inglehart, M.R. General dentists and special needs patients: does dental education matter? Journal of Dental Education. 2005;69(10):1107-15.

31.Subar, P.P., Chávez, E.M.E., Miles, J.J., Wong, A.A., Glassman, P.P., Labarre, E.E. Pre- and postdoctoral dental education compared to practice patterns in special care dentistry. Journal of Dental Education [Internet]. 2012;76(12):1623-8.

32. Ahmad, M.S., Razakm I.A., Borromeom G.L. Special Needs Dentistry: perception, attitudes and educational experience of Malaysian dental students. Eur J Dent Educ. 2014;19(1):44-52.

33. Watkins, C., Ettingerm R.L., Cowenm H., Qianm F., Dawsonm D.V. lowa dentists' involvement in care for patients who are homebound. Spec Care Dentist. 2012;32(6):251-8. 
34. Gizanim, S., Kandiloroum, H., Kavvadiam, K., Tzoutzas, J. Oral health care provided by Greek dentists to persons with physical and/or intellectual impairment. Spec Care Dentist. 2014;34(2):70-6

35. Chávez, E.M., Subarm P.E., Milesm J., Wongm A., Labarrem E.E., Glassmanm P. Perceptions of predoctoral dental education and practice patterns in special care dentistry. Journal of Dental Education. 2011;75(6):726-32.

36. Dougall, A., Fiske, J. Access to special care dentistry, part 1. Access. British Dental Journal. 2008;204(11):605-16.

37. Akbarali, N., Boyle, C., Newton, T. Appropriate referrals to special care dentistry- a retrospective study. Journal of Disability and Oral Health 2009;142-50.

38. Ahmad, M.S., Razak, I.A., Borromeo, G.L. Undergraduate education in special needs dentistry in Malaysian and Australian dental schools. Journal of Dental Education. 2014;78(8):1154-61.

License Information: This work is licensed under a Creative Commons Attribution 